\title{
Survival impact of lung transplantation for
} COPD

\author{
S. Lahzami*, P.O. Bridevaux ${ }^{\#}$, P.M. Soccal ${ }^{\#,}$, J. Wellinger ${ }^{+}$, J.H. Robert ${ }^{\dagger}$, \\ H.B. Ris ${ }^{+}$and J.D. Aubert ${ }^{*}, \S$
}

ABSTRACT: Chronic obstructive pulmonary disease (COPD) is the primary indication for lung transplantation (LTX), but survival benefit is still under debate. We analysed the survival impact of LTX in COPD with a new approach, using the BODE (body mass index, airway obstruction, dyspnoea, exercise capacity) index.

We retrospectively reviewed 54 consecutive lung transplants performed for COPD. The pretransplant BODE score was calculated for each patient and a predicted survival was derived from the survival functions of the original BODE index validation cohort. Predicted and observed posttransplant survival was then compared.

In the subgroups with a BODE score $\geqslant 7$ and $<7$, a majority of patients $(66 \%$ and $69 \%$, respectively) lived for longer after LTX than predicted by their individual BODE index. The median survival was significantly improved in the entire cohort and in the subgroup with a BODE score $\geqslant 7$. 4 yrs after LTx a survival benefit was only apparent in patients with a pre-transplant BODE score of $\geqslant 7$.

In conclusion, while a majority of COPD patients had an individual survival benefit from LTX regardless of their pre-transplant BODE score, a global survival benefit was seen only in patients with more severe disease. This supports the use of the BODE index as a selection criteria for LTX candidates.

KEYWORDS: BODE index, chronic obstructive pulmonary disease, lung transplantation, patient selection, survival analysis

hronic obstructive pulmonary disease (COPD) is a leading cause of morbidity and mortality around the world, making it one of the major challenges for the healthcare community. Medical treatment, including smoking cessation, bronchodilators, oxygen administration, adequate diet and pulmonary rehabilitation, remains the primary management of COPD. However, these are often insufficient in patients with advanced disease. For selected patients, surgical options include bullectomy, lung volume reduction surgery and lung transplantation (LTx) [1]. LTx for COPD was first reported in 1970, but has only been used on a larger scale since the late 1980 s, coinciding with the improvement of the surgical technique and immunosuppressive therapy. COPD is presently the main indication for LTx, accounting for more than one third of the procedures [2].

Although lung transplantation for COPD improves lung function [3], exercise capacity [4] and quality of life [5], controversy remains regarding survival benefit. Studies conducted to date, whether showing a survival benefit [6-8] or not $[9,10]$, have compared the survival of patients who underwent transplantation with patients remaining on the waiting list. Despite having used different approaches to take into account differences between patients on waiting lists and those who underwent the procedure, bias cannot be excluded.

In COPD patients, several univariate survival prognostic factors have been identified [11, 12]. However, the pathophysiology of COPD is complex and none of these factors alone is an accurate predictor of the survival of COPD patients. In 2004, from a large multicenter cohort of COPD patients CELLI et al. [13] identified a combination of four variables which showed a strong association with survival in COPD: bodymass index (B); airflow obstruction $(\mathrm{O})$; dyspnoea (D); and exercise capacity (E). These variables were used to create the BODE index, a multidimensional scoring system that was shown to be a better predictor of survival than the spirometric staging system developed by the American Thoracic Society [14]. The BODE index assigns a score from 0 to 10 , with a higher score indicating
AFFILIATIONS

*Service de Pneumologie,

${ }^{+}$Service de Chirurgie Thoracique,

${ }^{\S}$ Centre de Transplantation

d'Organes, Centre Hospitalier

Universitaire Vaudois, Université de Lausanne, Lausanne,

${ }^{\#}$ Service de Pneumologie, and

"Clinique de Chirurgie Thoracique, Hôpitaux Universitaires de Genève,

Geneva, Switzerland.

CORRESPONDENCE

J.D. Aubert

Service de Pneumologie et Centre de Transplantation d'Organes

Centre Hospitalier Universitaire

Vaudois

Rue du Bugnon 46

CH-1011 Lausanne

Switzerland

E-mail: John-David.Aubert@chuv.ch

Received:

June 032009

Accepted after revision:

Nov 262009

First published online:

Dec 082009 
more severe disease and predicting a poorer outcome. Thus, the BODE index offers an opportunity to evaluate the natural history of COPD patients enrolled in a lung transplantation programme by using criteria that are free of the selection bias introduced by the decisions of the transplantation team. This was recently emphasised by the introduction of a BODE score $\geqslant 7$ as a new recommended transplant criteria for COPD patients [15].

The primary goal of our study was to analyse the survival impact of lung transplantation in end-stage COPD patients, by comparing the effective post-transplant survival with the survival predicted by the BODE index as measured during pre-transplant clinical evaluation. The results were then applied to refine the selection criteria of LTx candidates.

\section{MATERIAL AND METHODS}

\section{Study subjects}

We retrospectively reviewed all consecutive subjects who underwent single or bilateral LTx (SLT and BLT, respectively) for COPD at Lausanne and Geneva University Hospitals (both Switzerland), from the start of the programme in 1993 until the end of 2007, with a follow-up until June 30, 2009. The diagnosis of COPD had been verified by a global assessment in every patient before listing, i.e. before the patient has an active status on the waiting list, with simultaneous assessment of suitability for LTx and potential cardiovascular, infectious or psychiatric contraindications to the procedure. Subjects with COPD related to $\alpha_{1}$-antitrypsin deficiency were excluded from the study.

\section{Data collection}

Demographic and clinical characteristics collected during the pre-transplant assessment period were recorded from the patient's chart, as well as the time to LTx and the type of procedure. The date of death, when applicable, was collected through centre-specific databases.

\section{BODE index calculation}

The pre-transplant BODE index score was calculated for each patient as described by CELLI et al. [13], using data obtained during the pre-transplant assessment: body mass index $\left(\mathrm{kg} \cdot \mathrm{m}^{-2}\right)$; post-bronchodilator forced expiratory volume in $1 \mathrm{~s}$ (FEV1) as percentage of the predicted value; score on the modified Medical Research Council (MMRC) dyspnoea scale; and 6-min walking distance (6MWD). Spirometry measurements and equations used to determine the predicted normal values for FEV1 were in accordance with the official statement of the European Respiratory Society for Standardized Lung Function Testing [16]. MMRC was collected or retrospectively evaluated from the patient's chart when necessary. 6-min walk tests without encouragement were performed. Missing 6MWD $(\mathrm{n}=3)$ were derived from the maximum oxygen uptake $\left(V^{\prime} \mathrm{O}_{2}\right.$,max $)$ measured during the pre-transplant assessment using the equations developed by CAHALIN et al. [17] in transplant candidates with end-stage lung disease. A $0 \mathrm{~m}$ $6 \mathrm{MWD}$ was assigned to the two patients who were not able to perform the 6-min walk test or the $V^{\prime} \mathrm{O}_{2}$, max test because of their respiratory condition.

\section{Data analysis}

We used the baseline survival function derived from the original BODE index cohort and provided by CELLI et al. [13]. The $95 \%$ confidence interval (CI) of the hazard ratio was used to calculate the lower boundary, intermediate and upper boundary of life expectancy for each BODE level. The predicted survival with lower and upper estimates of each patient was then individually calculated as derived from their BODE score, and compared to their effective post-transplant survival. Patients who were still alive at the end of the followup but had not yet achieved the upper boundary of their predicted survival were excluded from the analysis as the survival impact of LTx could not be evaluated. The survival effect of the procedure on the entire cohort was then assessed by comparing the predicted survival with the effective survival, and by determining the number of patients who individually benefited from the procedure. The same analysis was repeated in the subgroups with a pre-transplant BODE score $<7$ and $\geqslant 7$. The survival after SLT versus BLT, and the difference between transplant periods were also analysed.

\section{Statistical analysis}

A paired t-test and Chi-squared test were used when appropriate to compare patient's characteristics. The survival effect of LTx on the entire cohort and on BODE score subgroups were assessed by a Wilcoxon signed ranks test. Kaplan-Meier survival estimates were used to describe the post-transplant survival of the entire cohort and log rank test was used to compare the survival between types and periods of transplant, and between pre-transplant BODE subgroups. Statistical analyses were performed with SPSS 17.0 for Windows (SPSS Inc. Chicago, IL, USA).

\section{RESULTS}

A total of 54 patients with COPD unrelated to $\alpha_{1}$-antitrypsin deficiency underwent LTx from June 1993 until the end of 2007. No COPD patients listed for transplantation died during the waiting period. $26(48 \%)$ procedures were performed at Geneva University Hospitals and 28 (52\%) in Lausanne, using a similar surgical and medical approach. 35 (65\%) patients underwent BLT whereas 19 (35\%) underwent SLT. At the end of the follow-up, 29 (54\%) patients were still alive. Six of these patients had not yet achieved the upper boundary of their predicted survival according to their pre-transplant BODE index and were excluded from survival analysis. The flow chart of the patients and life status at the end of the follow-up is shown in figure 1 .

The cohort included a patient with a low BODE score of 2 , where the estimated half-life could not be calculated according to the data from the BODE original survival function [13]. Instead, the half-life was conservatively estimated according to the survival curve of the cohort of COPD patients described by MARTINEZ et al. [18]. One patient was re-transplanted during the study period and his survival time was calculated from the first LTx to death. Baseline characteristics of the transplanted patients are shown in table 1. Excluded patients did not differ significantly from the cohort used for survival analysis with respect to baseline characteristics. The mean \pm SD follow-up was $5.7 \pm 4.5$ yrs. 


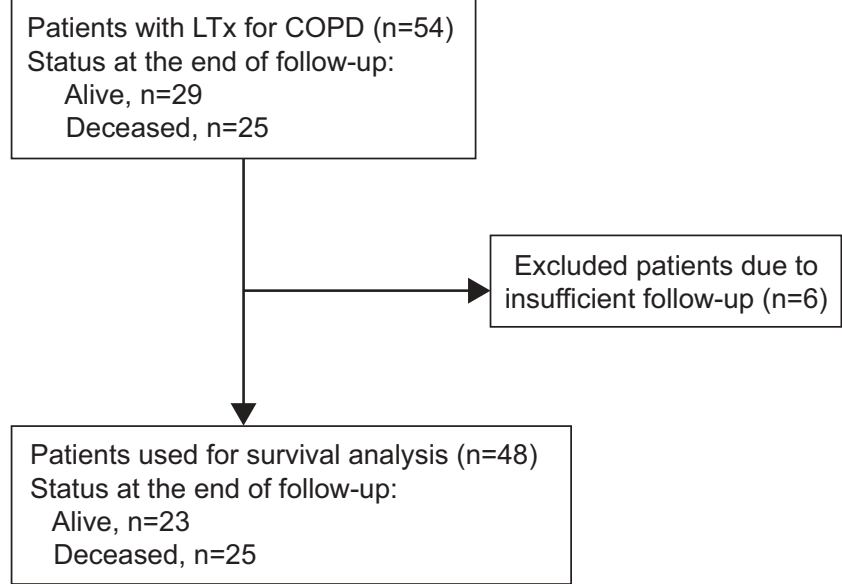

FIGURE 1. Flow-chart of the patients and life status at the end of the follow-up. LTx: lung transplantation; COPD: chronic obstructive pulmonary disease.

The comparison between observed post-transplant survival and expected survival according to pre-transplant BODE index is shown in table 2. For the whole cohort, the median survival was significantly improved after LTx. This survival benefit was seen in the subgroup with a BODE score $\geqslant 7$, but not in the subgroup with a BODE score $<7$, although a trend toward better survival with LTx was present. An individual survival benefit was seen in two thirds of the LTx recipients, regardless of their BODE score subgroup. The detailed survival loss or gain for each patient is shown in figure 2. It appears that a majority of patients lived much longer than expected while others, mainly in the BODE score $<7$ subgroup, experienced a potential survival loss.

A sensitivity analysis on the effect of the exclusion of six patients from the survival analysis was performed. A pessimistic, intermediate or optimistic survival was assigned to each of these excluded patients using the quartile 1 , median and quartile 3, respectively, of the survival observed in the other patients who reached at least the same post-transplant survival. These six patients were then included in the analysis. In the entire cohort $(n=54)$, the median post-transplant survival (pessimistic: 5.4 yrs; intermediate: 6.3 yrs; optimistic: 6.3 yrs) was significantly higher than the expected survival (lower boundary: 2.8 yrs; intermediate: 3.5 yrs; upper boundary: $4.2 \mathrm{yrs})$ in all cases. In the BODE $\geqslant 7$ subgroup $(n=35+2)$, the significant survival benefit of LTx was strengthened when compared to the lower boundary and intermediate expected survivals, and there was a trend towards benefit when compared to the upper boundary of predicted survival. The BODE $<7$ subgroup $(n=13+4)$ had a significant survival benefit from LTx only when compared to the lower boundary of the expected survival.

We found no significant survival difference between SLT and BLT subgroups, which had no between group pre-transplant differences. The period of transplant (1993-1999 versus 20002007) was not associated with a survival difference.

The Kaplan-Meier post-transplant survival was not different between pre-transplant BODE score subgroups (fig. 3). This allowed us to compare the predicted survival at each step of

\begin{tabular}{|c|c|c|c|c|}
\hline & \multirow[t]{2}{*}{ All } & \multicolumn{2}{|c|}{ BODE score } & \multirow[t]{2}{*}{ p-value ${ }^{\#}$} \\
\hline & & $<7$ & $\geqslant 7$ & \\
\hline Subjects & 48 & 13 & 35 & \\
\hline Male & $30(63)$ & $8(62)$ & $22(63)$ & 0.93 \\
\hline Age at LTx yrs & $55 \pm 6$ & $54 \pm 5$ & $56 \pm 6$ & 0.41 \\
\hline $\mathrm{BMI} \mathbf{k g} \cdot \mathrm{m}^{-2}$ & $22.4 \pm 4.2$ & $23.0 \pm 4.1$ & $22.2 \pm 4.3$ & 0.53 \\
\hline FEV $_{1} \%$ pred & $23 \pm 7$ & $29 \pm 8$ & $22 \pm 4$ & $<0.001$ \\
\hline \multicolumn{5}{|l|}{ MMRC dyspnoea scale } \\
\hline Class 2 & 5 & 5 & 0 & $<0.001$ \\
\hline Class 3 & 28 & 8 & 20 & \\
\hline Class 4 & 15 & 0 & 15 & \\
\hline 6MWD m & $242 \pm 121$ & $358 \pm 106$ & $199 \pm 97$ & $<0.001$ \\
\hline BODE index & $7.2 \pm 1.5$ & $5.3 \pm 1.2$ & $7.9 \pm 1.0$ & $<0.001$ \\
\hline Time on waiting list months & $6 \pm 4$ & $5 \pm 4$ & $7 \pm 4$ & 0.09 \\
\hline Bilateral lung transplantation & $30(63)$ & $8(62)$ & $22(63)$ & 0.93 \\
\hline \multicolumn{5}{|l|}{ Transplant period } \\
\hline 1993-1999 & 22 & 7 & 15 & 0.50 \\
\hline 2000-2007 & 26 & 6 & 20 & \\
\hline
\end{tabular}

Data are presented as $n, n(\%)$ or mean $\pm S D$, unless otherwise stated. BODE: body mass index, airway obstruction, dyspnoea, exercise capacity; LTx: lung transplantation; BMl: body mass index; FEV1: forced expiratory volume in $1 \mathrm{~s}$; \% pred: \% predicted; MMRC: Modified Medical Research Council; 6MWD: 6 min walk distance. \#: comparisons between BODE score $<7$ and BODE score $\geqslant 7$ subgroups

the BODE index with the effective Kaplan-Meier posttransplant survival of the entire cohort (table 3). This theoretical analysis showed that 4 yrs after LTx the survival benefit is limited to patients with a pre-transplant BODE score $\geqslant 7$.

\section{DISCUSSION}

This study showed a significant survival benefit of LTx in our cohort of COPD patients, with a median survival time significantly higher than expected prior to transplant. Considering that almost half of the patients were still alive at the end of the follow-up (fig. 1) these results are particularly relevant, as the importance of the survival benefit may have been underestimated due to a limited follow-up period. Moreover, a majority of patients had an individual benefit from the intervention in terms of survival.

These results support two previous studies that have shown a global survival benefit after 260 days [6] and 369 days [7], as well as a recent complex statistical simulation on the United Network for Organ Sharing database which showed a survival benefit in a majority of transplanted patients [8]. Two other studies did not demonstrate a survival benefit after 48 months [9] and 24 months [10] of follow-up, but the follow-up time of the latter was too short to allow meaningful comparisons.

Methodologically, we used the pre-transplant BODE index to predict a theoretical survival at time of LTx. In contrast, the five studies published to date compared, either directly or with a statistical model, the survival of transplanted patients with lung recipient candidates who remain on the waiting list. 


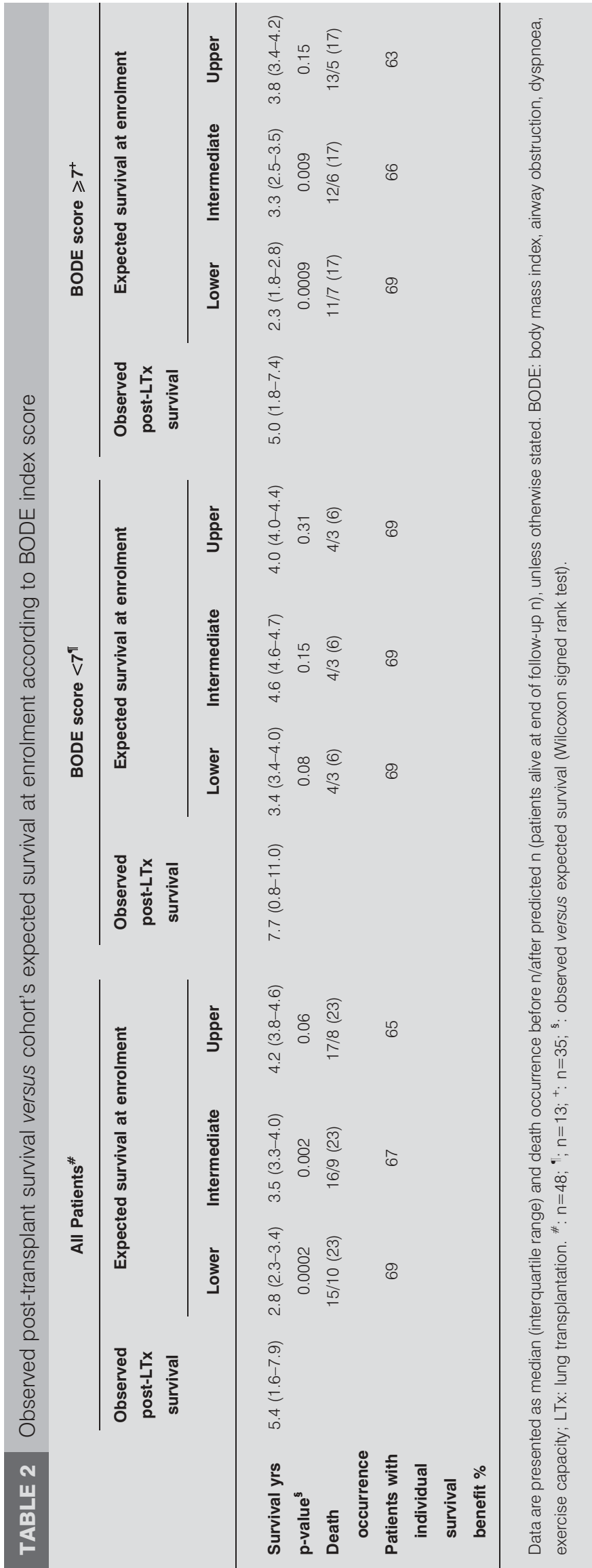

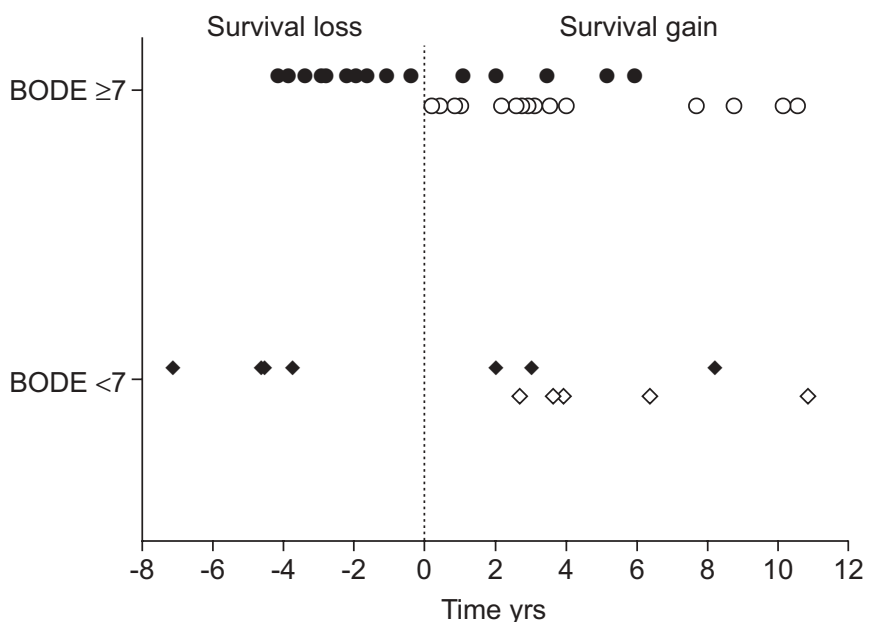

FIGURE 2. Individual survival impact of lung transplantation of each patient. Individual survival loss or gain was obtained for each patient by comparing the observed post-transplant survival with the $95 \%$ confidence interval upper boundary of predicted survival according to pre-transplant BODE score (body mass index, airway obstruction, dyspnoea, exercise capacity). Patients are separated into two subgroups based on their pre-transplant BODE score: BODE $\geqslant 7(n=35 ; \bullet, 0)$ and BODE $<7(n=13 ; \diamond, \diamond)$. Patients still alive at the end of the follow-up $(\diamond, 0)$ are presented differently to highlight their potentially longer survival gain. deceased at the end of follow-up.

This method of comparing survival is susceptible to potential bias against LTx. Indeed, the initial assumption is that all patients on the waiting list need a lung transplant at the time of listing. However, considering the usual duration of the waiting time (often beyond 2 yrs) [6, 19], transplant centres may register their patients early [20], which would improve the survival rate of the waiting list population. Furthermore, if patients on the waiting list are good candidates for transplantation at the time of listing, progression of the disease and ageing during waiting time makes them potentially worse candidates as older recipients have a significantly worse survival rate [2].

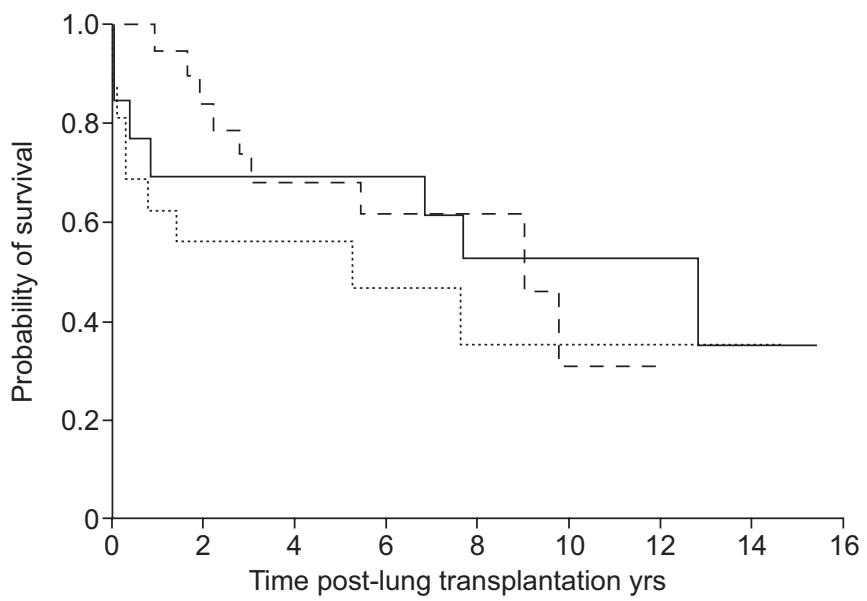

FIGURE 3. Observed post-transplant survival: difference between pre-transplant BODE (body mass index, airway obstruction, dyspnoea, exercise capacity)

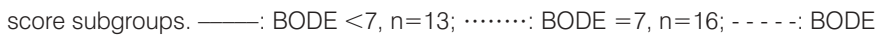
$>7, n=19 . p=0.62$. 


\begin{tabular}{|c|c|c|c|c|c|c|c|}
\hline 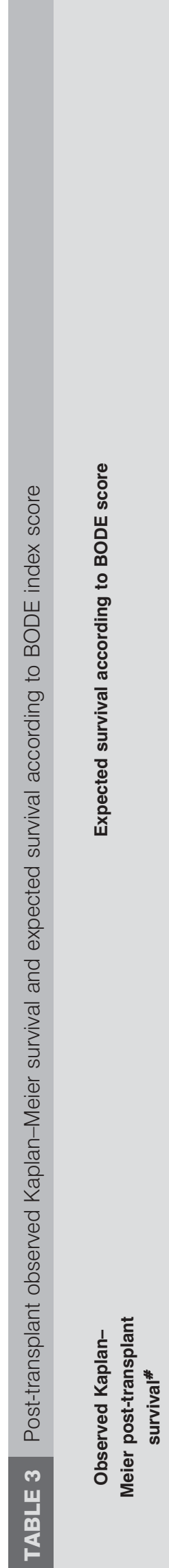 & 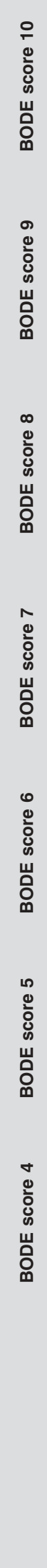 & 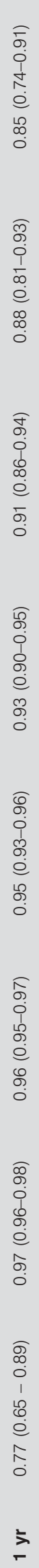 & $\begin{array}{l}\widetilde{0} \\
\infty \\
0 \\
0 \\
0 \\
0 \\
0 \\
0 \\
0 \\
0 \\
0 \\
\\
0 \\
\widehat{0} \\
0 \\
0 \\
0 \\
0 \\
0 \\
0 \\
0 \\
0 \\
0\end{array}$ & 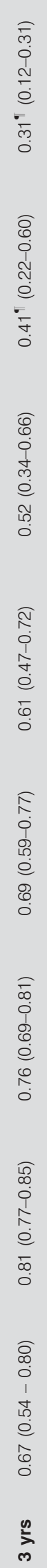 & 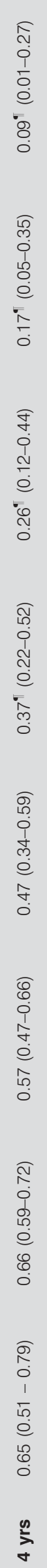 & 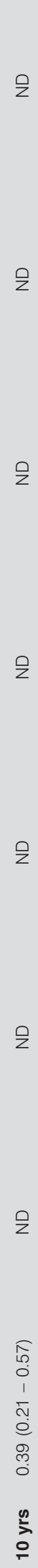 & 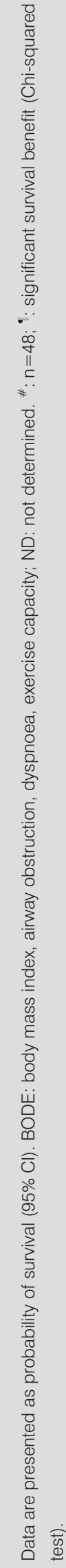 \\
\hline
\end{tabular}

In contrast, high-risk patients have an increased probability of dying while on the waiting list compared with low-risk patients who can survive long enough to undergo LTx. This could bias the results in favour of LTx. The different type of care received by patients on the waiting list compared to those not considered or denied for LTx is another probable major potential bias. By assigning a BODE score-based predicted survival time, postLTx survival can be compared with the predicted "natural" survival of the same patients, thus avoiding such risk of bias.

The post-LTx survival rates of our cohort, $77 \%$ at $1 \mathrm{yr}, 71 \%$ at $2 \mathrm{yrs}$ and $65 \%$ at $4 \mathrm{yrs}$, are in accordance with previous studies in COPD patients [2, 21-23]. The short waiting time ( $6 \pm 4$ months) and the absence of death while on the waiting list in our cohort may differ from other transplantation centres. However, our centres apply standard surgical and medical procedures, and the model used in this study is independent of any waiting list consideration. Therefore, our findings are applicable to other LTx centres.

Survival benefit is not the sole criteria to consider when evaluating the benefit of LTx in COPD patients, as LTx leads to a dramatic improvement in quality of life [5]. However, owing to the chronic shortage of lung donors, it is well accepted that LTx for COPD patients should be limited to a subset of those having the worst survival probability without intervention.

The presence of an FEV1 $<25 \%$ pred, an arterial carbon dioxide tension $\geqslant 55 \mathrm{mmHg}(7.3 \mathrm{kPa})$ or pulmonary arterial hypertension with progressive deterioration has been used for many years as the standard guidelines for the selection of COPD lung transplant candidates [24]. NATHAN et al. [25] were the first to recommend the additional use of the BODE index, and proposed a BODE score $\geqslant 7$ as a new transplant criteria. In 2006, the International Society of Heart and Lung Transplantation formalised the use of the BODE score in the guidelines for the selection of lung transplant candidates [15], by adding a BODE index score $\geqslant 7$ for transplantation and $\geqslant 5$ for referral.

We found that the post-transplant mortality risk did not depend on the pre-operative BODE index. Based on the observed survival in our cohort, the theoretical comparison with the survival predicted by each score of the BODE index showed a significant benefit at 4 yrs only with a pre-transplant BODE score $\geqslant 7$. Moreover, in our cohort, we found that LTx improved the median survival only in the subgroup with a BODE score $\geqslant 7$. In the subgroup with a BODE score $<7$, the mortality related to the intervention is higher than the longterm expected benefit. Thus, these patients should not be transplanted at this stage of the disease or the indication should at least be carefully re-examined. Our results highlight the need for the BODE index to become part of all pretransplant assessments in COPD patients and provide support to the official recommendation to use a cut-off BODE score of 7 as transplant criteria.

Our cohort's baseline BODE scores distribution was significantly higher than in the original BODE index validation cohort [13] (fig 4), reflecting the severity of COPD. The BODE index has not been specifically validated in a population listed for transplantation such as our cohort, but several factors suggest that this does not contraindicate its use. Not only did 
all our patients meet the inclusion criteria of CELLI et al. [13] but almost $50 \%$ of the BODE index validation cohort had BODE scores $\geqslant 5$ and, therefore, could have been referred for LTx as presently recommended [15]. More importantly, the BODE index has already proven its ability to predict mortality in severe COPD patients included in the National Emphysema Treatment Trial study [18]. These patients were probably selected and followed as closely as patients listed for LTx. Moreover, the use of $95 \% \mathrm{CI}$ of the survival predicted by the BODE index probably accounts for most of the differences between patients which are not evaluated by the BODE index, such as age, presence of pulmonary hypertension or other comorbidities. Nevertheless, the possibility that, due to a positive selection bias, the BODE index might be less accurate at predicting mortality in a cohort listed for transplantation has to be considered when interpreting the results of our study.

The determination of BODE index scores from data collected during pre-transplant assessment is another limitation that should be recognised. Consequently, the calculated theoretical survival was not identical to the one at the time of LTx. However, considering the short waiting time ( $6 \pm 4$ months), a significant worsening of patients conditions during this time is unlikely. The absence of deaths during this period reinforces this hypothesis. In any case, it would result in an underestimation of BODE index scores at the time of transplant and, thus, an overestimation of the predicted survival time. Consequently, we would have found a higher survival benefit of LTx.

In summary, the results of this study showed a significant survival benefit of LTx in our cohort of COPD patients. Importantly, not only was the median survival improved with LTx but a significant majority of patients had an individual survival benefit from this procedure. Although the latter was independent from pre-transplant BODE score, the former was seen in the entire cohort and in the subgroup with a BODE score $\geqslant 7$, but not in the subset of patients with a BODE score $<7$. Moreover, in a theoretical analysis, we found that 4 yrs after LTx a survival benefit could only be expected in the patients with a pre-transplant BODE score $\geqslant 7$. For those with

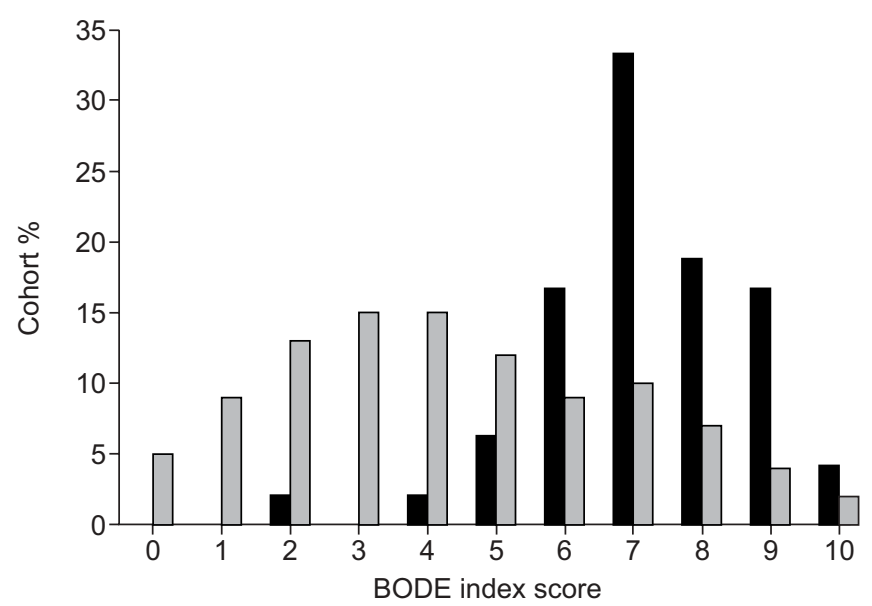

FIGURE 4. BODE (body mass index, airway obstruction, dyspnoea, exercise capacity) index repartition: studied transplanted cohort ( $\mathbf{\square})$ versus original BODE index validation cohort $(\square)$. Data modified from [13] low BODE scores, the risk of the procedure outweighs the survival benefit. These results support the current recommendation to use a BODE score $\geqslant 7$ as transplant criteria for patients with COPD.

\section{STATEMENT OF INTEREST}

A statement of interest for J.D. Aubert can be found at www.erj. ersjournals.com $/ \mathrm{misc} /$ statements.dtl

\section{ACKNOWLEDGEMENTS}

We are grateful to B.R. Celli and colleagues who provided the original BODE index validation cohort's baseline survival function, W. Xuan for his statistical advice, and all present and former members of the lung transplant units of Lausanne and Geneva University Hospitals (both Switzerland) who have assisted in the care of these patients.

\section{REFERENCES}

1 Martinez FJ, Chang A. Surgical therapy for chronic obstructive pulmonary disease. Semin Respir Crit Care Med 2005; 26: 167-191.

2 Christie JD, Edwards LB, Aurora P, et al. Registry of the International Society for Heart and Lung Transplantation: twentyfifth official adult lung and heart/lung transplantation report. J Heart Lung Transplant 2008; 27: 957-969.

3 Mason DP, Rajeswaran J, Murthy SC, et al. Spirometry after transplantation: how much better are two lungs than one. Ann Thorac Surg 2008; 85: 1193-1201.

4 Pochettino A, Kotloff RM, Rosengard BR, et al. Bilateral versus single lung transplantation for chronic obstructive pulmonary disease: intermediate-term results. Ann Thorac Surg 2000; 70: 1813-1819.

5 Rodrigue JR, Baz MA, Kanasky WF Jr, et al. Does lung transplantation improve health-related quality of life? The University of Florida experience. J Heart Lung Transplant 2005; 24: 755-763.

6 De Meester J, Smits JMA, Persijn GG, et al. Listing for lung transplantation: life expectancy and transplant effect, stratified by type of end-stage lung disease, the Eurotransplant experience. J Heart Lung Transplant 2001; 20: 518-524.

7 Charman SC, Sharples LD, McNeil KD, et al. Assessment of survival benefit after lung transplantation by patient diagnosis. J Heart Lung Transplant 2002; 21: 226-232.

8 Thabut G, Ravaud P, Christie JD, et al. Determinants of the survival benefit of lung transplantation in patients with chronic obstructive pulmonary disease. Am J Respir Crit Care Med 2008; 177: $1156-1163$.

9 Stavem K, Bjortuft O, Borgan O, et al. Lung transplantation in patients with chronic obstructive pulmonary disease in a national cohort is without obvious survival benefit. J Heart Lung Transplant 2006; 25: 75-84.

10 Hosenpud JD, Bennet LE, Keck BM, et al. Effect of diagnosis on survival benefit of lung transplantation for end-stage lung disease. Lancet 1998; 351: 24-27.

11 Dolan S, Varkey B. Prognostic factors in chronic obstructive pulmonary disease. Curr Opin Pulm Med 2005; 11: 149-152.

12 Martinez FJ, Kotloff R. Prognostication in chronic obstructive pulmonary disease: implications for lung transplantation. Semin Respir Crit Care Med 2001; 22: 489-498.

13 Celli BR, Cote CG, Marin JM, et al. The body-mass-index, airflow obstruction, dyspnea, and exercise capacity index in chronic obstructive pulmonary disease. N Engl J Med 2004; 350: 1005-1012.

14 Standards for the diagnosis and care of patients with chronic obstructive pulmonary disease. American Thoracic Society. Am J Resp Crit Care Med 1995; 152: S77-S120.

15 Orens JB, Estenne M, Arcasoy S, et al. International guidelines for the selection of lung transplant candidates: 2006 update - a consensus report from the Pulmonary Scientific Council Of The 
International Society For Heart And Lung Transplantation. J Heart Lung Transplant 2006; 25: 745-755.

16 Quanjer PH, Tammeling GJ, Cotes JE, et al. Lung volumes and forced ventilatory flows. Report Working Party Standardization of Lung Function Tests, European Community for Steel and Coal. Official Statement of the European Respiratory Society. Eur Respir J 1993; 6: Suppl. 16, 5S-40S.

17 Cahalin L, Pappagianopoulos P, Prevost S, et al. The relationship of the 6-min walk test to maximal oxygen consumption in transplant candidates with end-stage lung disease. Chest 1995; 108: 452-459.

18 Martinez FJ, Foster G, Curtis JL, et al. Predictors of mortality in patients with emphysema and severe airflow obstruction. Am J Resp Crit Care Med 2006; 173: 1326-1334.

19 Trulock EP. Lung and heart-lung transplantation: overview of results. Semin Respir Crit Care Med 2001; 22: 479-488.

20 Travaline JM, Cordova FC, Furukawa S, et al. Discrepancy between severity of lung impairment and seniority on the lung transplantation list. Transplant Proc 2004; 36: 3156-3160.
21 Thabut G, Christie JD, Ravaud P, et al. Survival after bilateral versus single lung transplantation for patients with chronic obstructive pulmonary disease: a retrospective analysis of registry data. Lancet 2008; 371: 744-751.

22 Günes A, Aboyoun CL, Morton JM, et al. Lung transplantation for chronic obstructive pulmonary disease at St Vincent's Hospital. Intern Med J 2006; 36: 5-11.

23 Cassivi SD, Meyers BF, Battafarano RJ, et al. Thirteen-year experience in lung transplantation for emphysema. Ann Thorac Surg 2002; 74: 1663-1669.

24 International guidelines for the selection of lung transplant candidates. The American Society for Transplant Physicians (ASTP)/American Thoracic Society (ATS)/European Respiratory Society (ERS)/International Society for Heart and Lung Transplantation (ISHLT). Am J Respir Crit Care Med 1998; 158: 335-339.

25 Nathan SD. Lung transplantation: disease-specific considerations for referral. Chest 2005; 127: 705-707. 